\section{卒 IOI}

We aopreciate the response to this publication feature - and welcome all contributions. Contributions may be sent to Phil Oshel, our Technical Ed. for at:

$\begin{array}{ll}\text { Mr. Phil Oshel } & \text { (609)833-2805 } \\ \text { PO Box 620068 } & \text { eMall: oshelGlerracom net } \\ \text { Middloton W/ } 53562 & \\ \text { (608)833-2885 } & \text { PLease note NEW address }\end{array}$

\section{Tips on Finding Grain Boundaries in the TEM}

I spent some time working on beta silicon nitride, and I found it very time consuming, because one needs to find orientations such that two gains are in orientations that can be imaged, while at the same time the grain boundary needs to be parallel to the beam. The paper by D.R. Clark (Ultramicroscopy 4 (1979)33-44) is useful.

What I found to be helpful was to first check the image of the boundary at lower magnifications (typically $\times 100,000$ ) in bright field mode and tilt about an axis parallel to the image of the boundary until I attained a relatively sharp "minimum" in the width of the boundary. This is relatively last, and it allows elimination of those grain boundaries that are way out of crientation (I was using a $30^{\circ}$ double till holder).

Once I had what seemed to be a suitable grain boundary. then at that point I would check the diffaction pattern to see if there were any Kikuchi lines (from either grain) that were parallel to the grain boundary and which corresponded to reflections that could be used for high resolution TEM imaging (l.e, reflections that had a large enough d-spacing). If that was the case, then I would tilt the salmple alang that Kikuchi line $(d$. e. maintaining that set of reflections for that particular grain) and at the same time check the diffaction pattern from the other gain until a suitable orientation was attained for that second gain. More often than not, I had to move on to another location and start the whole aperation again until all three elements (grain 1 , grain 2 and the grain boundary) had the proper orientation.

In most instances, my images consisted of two beam conditions, but that was sufficient to resolve the grain boundaries which were typically about 1 to $4 \mathrm{~nm}$ wide. In one afternoon session I could probably end up with three to five suitable boundaries (plus coffee breaks)

Jordi Marti, Allied Sighal

\section{A Hint for Reducing Background for Immuno-TEM}

Non-specific background staining is occasionally a problem with some antibodies and labeling systems. If nomal washing fails to reduce the background you might want to ty using a high salt buffer for washing. Phosphate buffered saline or any other recipe with $\mathrm{NaCl}$ in it, is probably around $150 \mathrm{mM}(0.9 \%)$. Try boasting the $\mathrm{NaCl}$ to $5 \mathrm{X}$ nomal. This would make it $750 \mathrm{mM}$, or $4.5 \%$ by weight.

If high salt buffer is used, remember to incubate the grids in a couple of changes of regular stength $(150 \mathrm{mM})$ saline before going to the next step, to get the salt back into the range of physiologic strength.

An example would be to wash the grids on five drops of high salt buffer for about 2 minutes each, followed by two dops of nomal salt buffer for about 2 minutes each. Use this routine after incubating on the primary antbody or after any incubation you suspect may be contributing to background (secondary antibody, colloidal gold, etc.).
I have not found a good explanation for why this works, although it probably changes the conformation of proteins, alters their overall charge, and makes them less likely to bind to the surface of the sectioned material.

High salt concentrations are often used in biochemisty to precipitale proteins out of solution and to wash chromatography columns, Something similar is probably happening on the surface of the section, j.e., only those antibody molecules which have bound specifically to antigenic sites are able to stay on the section in the presence of high salt concentrations.

Robert (Rob) Chiovefli. E. Licht Company

\section{A Solution for Cultured Lymphocytes Missing Membranes After Preparation for TEM}

This is a very rapid process.

The cell suspension is processed in centrifuge lubes, Spin cells at $2500 \mathrm{rpm}$ for one minute, pipette of culture medium, and replace with $2.5 \%$ glutaraldehyde in $0.1 \mathrm{M}$ Na cacodylate and resuspend.

Immediately place tube in centrifuge and spin for 1 minute at 2000 rpm.

Each solution was changed, cells resuspended and spun immediately, therefore each solution was used for about 1,5 minutes.

Buffer wash with $0.1 \mathrm{M}$ Na cacodylate

$2 \% \mathrm{OsO}_{4}$ in same buffer

buffer wash (once)

$10 \%$ ethanol

$5 \%$ aculueous uranyl acetate

$50 \%$ ethanol

$75 \%$ ethanol

$100 \%$ ethanal

into Spurr resin in Eppendorf centifuge tubes and straight into oven: polymerize at $60^{\circ} \mathrm{C}$.

I processed literally as fast as possible. An earlier attempt using 5 minutes at each step was an improvement but not good enough.

I suspect that the cell line is unusual in needing this short a time, but it's worth trying for other cells.

Chris Gilpin, Manchester University

\section{If Your Older Model ISI/Topcon SEM Blows Transistors...}

Some ofder IsUTopcon SEMs regularly blow the transistors in the box on top of the HT tank. We think we have been able to identify and solve the problem.

The circuit inside the tank an the bias and HT side that has the 4700 pF and $2200 \mathrm{pF}$ caps on it, is to filter out the back electromagnetic field from the high voltage side of the tank. We found that these same capacitors go soft, and so leak that voltage back up into the diver tansistors on top of the tank. This is what causes them to blow.

It is quite simple to monitor this. If you measure the collectors of these tansistors, you should measure waweforms with a peak voltage not exceeding $400 \mathrm{~V}$ on any of the tansistors (the actual voltage will vary with bias settings, filament cument and accelerating voltage). Should you find that the voltage is close to this voltage or over, then replace the relevant capacitors in the tank $(\mathrm{C} 20, \mathrm{C21}, \mathrm{C4}, \mathrm{CS}$, etc., $4700 \mathrm{pF} 2.5 \mathrm{kV}$ and 2200 $\mathrm{pF} 4 \mathrm{kV}$ and $0.1 \mu \mathrm{F} 630 \mathrm{v}$ ). This will solve the problem.

This is applicable to quite a range of their models, from the $\mathbf{S \times 3 0}$. SX40, SS60, DS130, WB6, and on to the newer series of ABT 55 and 60 . These are the only models we have here in South Africa. This tip may apply to more models, but we have nat seen them.

Luc Harmsen, Anaspec, South Africa 